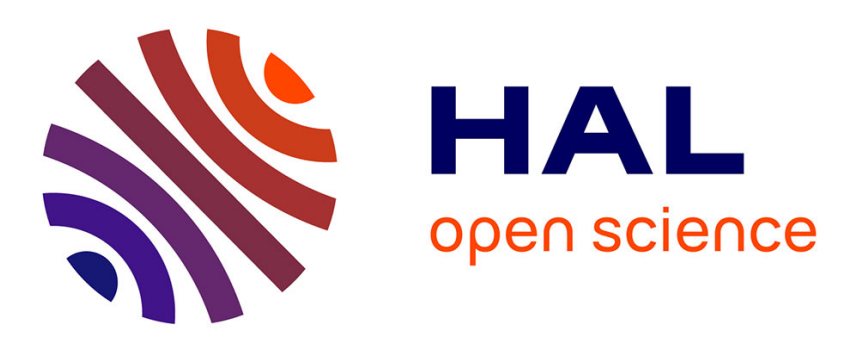

\title{
On the stability of the quasi-onedimensional metallic phase in magnetic fields against the spin density wave formation
}

\author{
L.P. Gor'Kov, A.G. Lebed'
}

\section{To cite this version:}

L.P. Gor'Kov, A.G. Lebed'. On the stability of the quasi-onedimensional metallic phase in magnetic fields against the spin density wave formation. Journal de Physique Lettres, 1984, 45 (9), pp.433-440. 10.1051/jphyslet:01984004509043300 . jpa-00232366

\section{HAL Id: jpa-00232366 https://hal.science/jpa-00232366}

Submitted on 1 Jan 1984

HAL is a multi-disciplinary open access archive for the deposit and dissemination of scientific research documents, whether they are published or not. The documents may come from teaching and research institutions in France or abroad, or from public or private research centers.
L'archive ouverte pluridisciplinaire HAL, est destinée au dépôt et à la diffusion de documents scientifiques de niveau recherche, publiés ou non, émanant des établissements d'enseignement et de recherche français ou étrangers, des laboratoires publics ou privés. 
Classification

Physics Abstracts

$75.50-71.30$

\title{
On the stability of the quasi-onedimensional metallic phase in magnetic fields against the spin density wave formation
}

\author{
L. P. Gor'kov and A. G. Lebed' \\ L.D. Landau Institute for Theoretical Physics, The USSR Academy of Sciences, \\ 117334 Moscow V-334 ul. Kosygina 2, U.S.S.R.
}

(Reçu le 24 janvier 1984, accepté le 16 mars 1984)

\begin{abstract}
Résumé. - Nous montrons que la stabilité d'un état à ondes de densité de spin d'un métal quasi unidimensionnel, à surface de Fermi ouverte, augmente sous champ magnétique. Ce phénomène peut conduire à une série de transitions sous champ. Nous suggérons que ce modèle peut être une interprétation du diagramme de phase des composés (TMTSF) ${ }_{2} \mathrm{X}$ sous champ magnétique.
\end{abstract}

\begin{abstract}
For a simple anisotropic metal model with two open nested Fermi surfaces, it is shown that the tendency to spin density wave formation increases when a magnetic field is applied, which could lead to a series of phase transitions as functions of the field value. The results are suggested as an explanation of the phase diagram of the (TMTSF) $)_{2} \mathrm{X}$ compounds.
\end{abstract}

In this letter we report an unexpected result, predicting in the framework of a certain theoretical model, the instability of the quasi-onedimensional (Q1D) metal state with respect to spin density wave (SDW) formation in a moderate magnetic field. It is in direct correspondence with the recent experimental discovery of the remarkable phase diagram in the (TMTSF $)_{2} \mathrm{X}$ compound family (see in Refs. [1-6]). A typical phase diagram in the ( $T, P, H)$-space for these materials, as has been obtained for $\mathrm{X}=\mathrm{PF}_{6}$ and $\mathrm{X}=\mathrm{ClO}_{4}$, is shown in figure 1 . The twice shaded part of the surface is the boundary between the anisotropic (Q1D) metal phase with quasiplanar open Fermi surfaces and the field-induced spin density wave (FISDW) state. This boundary is marked with the so-called " onset " or threshold field $H_{0}$ which is both temperature- and pressure-dependent [3, 4]. The magnetic nature of the FISDW state is tested in NMR measurements $[5,6]$, the Shubnikov-de Haas $(\mathrm{SdH})$ oscillations, observed in the magnetic field $H$ exceeding the onset field $H>H_{0}[3,4]$, have enabled one to conclude the semi-metallic character of this state. However some unusual features of these oscillations $[1,4]$ and especially the most recent observation [7, 8] of the step-like Hall voltage behaviour with the field in (TMTSF) ${ }_{2} \mathrm{ClO}_{4}$, in particular, confirming the fact that the Fermi surface is open at $H<H_{0}$, have led to the idea [7] that these fields $H_{k}$ at which the " $\mathrm{SdH}$ » oscillations occur, could actually correspond to some cascade of phąse subtransitions in the FISDW state. (The alternative explanation [7] of the observed Hall steps in terms of the quantized Hall conductivity is not discussed here, since the longitudinal resistivity does not testify to any decrease at these fields.)

Low values for all typical parameters $\left(T_{\text {SDw }} \sim 10 \mathrm{~K}, P \sim 10 \mathrm{kbar}, H \sim 50 \mathrm{kOe}\right)$, characterizing the phenomena reflected by the phase diagram in figure 1 , demand an explanation which 


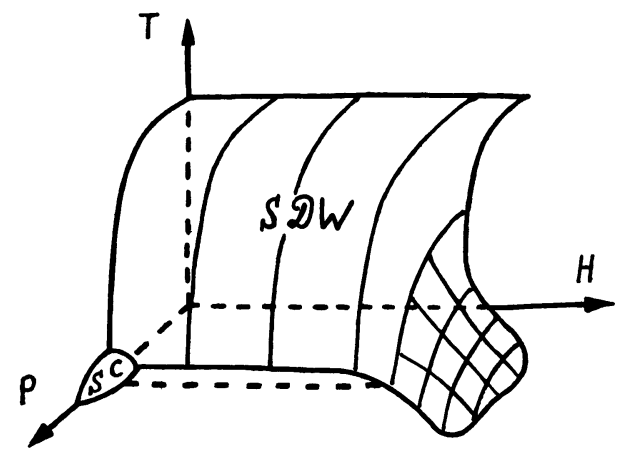

Fig. 1. - Phase diagram of the (TMTSF $)_{2} \mathrm{X}$ salts in the $(P, T, H)$-space.

would permit one to forget high temperature properties of these compounds due to their original Q1D structure. If this point of view is accepted the simplest explanation of the appearance of the antiferromagnetic state in these materials lies in the Overhauser mechanism of SDW formation $\left({ }^{1}\right)$ based on the Keldysh-Kopaev model [9] with the electron spectrum property :

$$
\varepsilon(\mathbf{p}+\mathbf{Q})=-\varepsilon(\mathbf{p})
$$

(here $\mathbf{Q}$ is the nesting vector characterizing the spin wave periodicity).

This explanation has first been suggested in [10] where the tight binding model has been used for the energy spectrum :

$$
\varepsilon(\mathbf{p})= \pm v\left(p_{\|} \mp k_{\mathrm{F}}\right)+t_{\perp}\left(\mathbf{p}_{\perp}\right)
$$

where the plus and minus correspond to the spectrum near the right-hand and left-hand quasiplanar sheets of the Fermi surface, respectively. In the nearest-neighbour interaction approximation [10] :

$$
t_{\perp}^{0}\left(\mathbf{p}_{\perp}\right)=t_{b^{*}} \cos \left(p_{b^{*}} b^{*}\right)+t_{c^{*}} \cos \left(p_{c^{*}} c^{*}\right)
$$

perfect nesting is achieved if

$$
\mathbf{Q}=\left(2 k_{\mathrm{F}}, \pi / b^{*}, \pi / c^{*}\right)
$$

which for the (TMTSF) ${ }_{2} \mathrm{X}$ family would correspond to the double lattice periods in all space directions. (In what follows we use the orthorhombic lattice model.) The 1D nesting vector

$$
\mathbf{Q}^{\prime}=\left(2 k_{\mathrm{F}}, 0,0\right)
$$

shall be discussed later, but at first glance this choice would not be compatible with the existence of the gap (dielectric state at $T=0$ ) in (TMTSF) ${ }_{2} \mathrm{PF}_{6}$ and with a fairly low $T_{\text {SDw }}$ at ambient pressure $(\sim 12 \mathrm{~K})$ and the estimated values of $t_{b^{*}} \sim 100 \mathrm{~K}$ [11]. An account of the next-neighbour interactions would slightly change the spectrum (2):

$$
t_{\perp}\left(\mathbf{p}_{\perp}\right)=t_{\perp}^{0}\left(\mathbf{p}_{\perp}\right)+t_{\perp}^{\prime}\left(\mathbf{p}_{\perp}\right)
$$

( $\left.{ }^{1}\right)$ We do not discuss here the reasons why the tendency to the SDW formation prevails over the CDW pairing in these materials taking merely the fact as given. 
and $t_{\perp}^{\prime}\left(\mathbf{p}_{\perp}\right)$ is now responsible for violations of perfect nesting of the Fermi surface sheets (3) and (4). As is known, the nesting pairing allows for some deviations from the perfect conditions if the value of $t_{\perp}^{\prime}$ does not considerably exceed the magnitude of $T_{0} \equiv T_{\text {SDw }}$ at $t_{\perp}^{\prime} \equiv 0$.

The key idea for the subsequent discussion is that, when the pressure is applied, the value of $t_{\perp}^{\prime}$ increases gradually suppressing the SDW state in accordance with figure 1 . (The change of $t_{\perp}^{0}$ does not violate the nesting conditions, we shall also not consider the narrow field interval for the superconductivity on the phase diagram).

These comments are equally relevant to both the SDW and CDW types of pairing. A magnetic field introduces distinction between the two behaviours. Whereas the antiferromagnetic type of pairing corresponds to the nesting vector between the Fermi surfaces for spins of opposite directions (the vector is not affected by the field), the CDW pairing takes place for spins of the same directions. As a result, instead of (1)

$$
\varepsilon(\mathbf{p}+\mathbf{Q})+\varepsilon(\mathbf{p})=2 \mu_{\mathrm{B}} H
$$

which points to the mechanism by which the applied field, in principle, inhibits the CDW pairing.

To conclude this short discussion, let us also mention that in most experimental environments the phase diagram of figure 1 corresponds to the geometry when the field is oriented in a parallel direction to the $c^{*}$-axis of the sample $\left(H / / c^{*}\right)$. These materials at low temperatures are actually more like layered compounds with the TMTSF-conductivity sheets in the $\left(a, b^{*}\right)$-plane. Correspondingly, it is also manifested in very small values $t_{c^{*}} \ll t_{b^{*}}$.

Thus, it is necessary to find a mechanism restoring in high magnetic fields the tendency to SDW pairing in the metallic phase (the respective pressure region is shown by the dotted line in Fig. 1). The physical idea is that an electron moving in $p$-space along one of the open Fermi surface sheets in direct space performs motion along the quasiclassical trajectory, determined by the momentum $\pm k_{\mathrm{F}}$ and this motion becomes limited and periodic in the perpendicular direction in the presence of the field due to the periodicity of the dispersion law $t_{\perp}\left(\mathbf{p}_{\perp}\right)$ in (2). This would increase the effective one-dimensionality of electron properties. To demonstrate this effect quantitatively, we shall consider the generalized susceptibility, $\chi(\mathbf{Q}, T, P, H) \equiv \chi(\mathbf{Q})$, describing the linear response of the system to the external staggered field :

$$
\hat{h}(\mathbf{Q})=\left(\hat{\sigma}_{y}\right)_{\alpha \beta} \exp (i \mathbf{Q}) \text {. }
$$

An expression for the susceptibility is easily obtained by summation of the standard « ladder » diagrams :

$$
\chi(\mathbf{Q})=\chi_{0}(\mathbf{Q}) /\left[1-\lambda \chi_{0}(\mathbf{Q})\right]
$$

(Here $\chi_{0}(\mathbf{Q})$ is the response for noninteracting electrons at given $(T, P, H), \lambda$ is an appropriate interaction constant [10].) As for $\chi_{0}(\mathbf{Q})$, it is more convenient to write it now in the mixed $\left(\omega, \mathbf{p}_{\perp}, x\right)$ representation :

$$
\chi_{0}(\mathbf{Q})=T \sum_{\omega_{n}} \int \frac{\mathrm{d} \mathbf{p}_{\perp}}{(2 \pi)^{2}} \int \mathrm{d} x^{\prime} g_{++}\left(i \omega_{n}, \mathbf{p}_{\perp} ; x, x^{\prime}\right) g_{--}\left(i \omega_{n}, \mathbf{p}_{\perp}-\mathbf{Q} ; x^{\prime}, x\right)
$$

where $g_{++}\left(g_{--}\right)$are slowly varying parts of the electron Green function near the right(left)-hand Fermi surface sheets. Thus

$$
G_{++}\left(i \omega_{n}, \mathbf{p}_{\perp} ; x, x^{\prime}\right)=\exp \left[i k_{\mathrm{F}}\left(x-x^{\prime}\right)\right] g_{++}\left(i \omega_{n}, \mathbf{p}_{\perp} ; x, x^{\prime}\right)
$$

Taking the geometry into account $\left(H / / c^{*}\right)$, substituting $\mathbf{p} \rightarrow \mathbf{p}-\frac{e}{c} \mathbf{A}$ into (8) and choosing the 
vector potential, $\mathbf{A}$, in the form $\mathbf{A}=(0, H x, 0)$, we arrive at the equation defining $g_{+}+$

$$
\left\{i \omega_{n}+i v \frac{\mathrm{d}}{\mathrm{d} x}-t_{\perp}\left(p_{b^{*}}-\frac{e H x}{c}, p_{c^{*}}\right)\right\} g_{++}\left(i \omega_{n}, \mathbf{p}_{\perp} ; x, x^{\prime}\right)=\delta\left(x-x^{\prime}\right)
$$

which is immediately integrated in the form

$$
g_{++}=\frac{\operatorname{sign} \omega_{n}}{i v} \exp \left\{-\frac{\omega_{n}\left(x-x^{\prime}\right)}{v}-\frac{i}{v} \int_{x^{\prime}}^{x} t_{\perp}\left(p_{b^{*}}-\frac{e H u}{c}, p_{c^{*}}\right) \mathrm{d} u\right\}
$$

at $\omega_{n}\left(x-x^{\prime}\right) / v>0$ (and is zero in the opposite case). The substitution of (9) into (8) (the expression for $g_{--}$results from (9) due to the change of the sign of $v$ ) shows that only the terms $t_{\perp}^{\prime}\left(\mathbf{p}_{\perp}\right)$ making the nesting imperfect are retained in equation (8). In what follows :

$$
t_{\perp}^{\prime}\left(\mathbf{p}_{\perp}\right)=2 t_{b^{*}}^{\prime} \cos \left(2 p_{b^{*}} b^{*}\right)+2 t_{c^{*}}^{\prime} \cos \left(2 p_{c^{*}} c^{*}\right) .
$$

Unlike (3), equation ( $\left.3^{\prime}\right)$ includes the next-neighbour interactions. By means of $\left(3^{\prime}\right)$, (8) can be rewritten explicitly. After averaging (8) over $\mathbf{p}_{\perp}$ and after subsequent simplifications the stability condition for the metallic phase, implying that the denominator in (7) is positive, finally becomes :

$$
\frac{1}{\zeta}-\int_{d}^{\infty} J_{0}\left[\frac{4 c t_{b^{*}}^{\prime}}{v e H b^{*}} \sin \left(\frac{b^{*} e H}{c} x\right)\right] J_{0}\left(\frac{4 t_{c^{*}}^{\prime}}{v} x\right) \frac{2 \pi T \mathrm{~d} x}{v \operatorname{sh}\left(\frac{2 \pi T x}{v}\right)} \geqslant 0
$$

(Here the dimensionless constant $\zeta=\frac{\lambda b^{*^{-1}} c^{*^{-1}}}{2 \pi v}$ and the low cutoff, $d$, were introduced. As usual, the cutoff will drop out from equation (10), if the relation $\zeta^{-1}=-\ln \frac{\pi T_{0} d}{v}$ is used, where $T_{0}$ is the transition temperature at $t_{b^{*}}^{\prime}=t_{c^{*}}^{\prime}=0$.)

The physical effect which has already been qualitatively explained above, is now evident from equations (9) and (10). In fact, at $H \neq 0$ the argument of the first Bessel function is now periodically dependent on $x$. If $t_{c^{*}}^{\prime} \ll t_{b^{*}}^{\prime}$, in the first approximation equation (10) transforms

$$
\frac{1}{\zeta}-\int_{d}^{\infty} J_{0}\left[\frac{4 c t_{b^{*}}^{\prime}}{v e H b^{*}} \sin \left(\frac{b^{*} e H}{c} x\right)\right] \frac{2 \pi T \mathrm{~d} x}{v \operatorname{sh}\left(\frac{2 \pi T x}{v}\right)} \geqslant 0
$$

A new feature of this expression, compared to the case $H=0$, is the appearance of the logarithmic divergence in the integral at large $x\left(x>c / e H b^{*}\right)$. Averaging over $x$ and using the relation

$$
\frac{1}{\pi} \int_{0}^{\pi} J_{0}(z \sin \varphi) \mathrm{d} \varphi=J_{0}^{2}(z / 2)
$$

one sees from $\left(10^{\prime}\right)$ and $(11)$ that $\left(\right.$ at $\left.t_{c^{*}}^{\prime}=0\right)$ the metallic phase would never be stable at zero temperature and finite $H$, except a discrete set, $H_{k}$, of certain values of the magnetic field, $H$, defined by the condition (11) :

$$
J_{0}\left(\frac{2 c t_{b^{*}}^{\prime}}{v e H b^{*}}\right)=0
$$


Bearing in mind that $2 t_{b^{*}}^{\prime}>\pi T_{0} / \gamma$ (i.e., at $H=0$ the normal metallic phase corresponds to the given pressure) and substituting the first zero of the Bessel function, one finds that the first point of this set, $H_{1}$, from the right-hand side (i.e., from the side of large fields) lies at

$$
H_{1} \lesssim \frac{\pi c T_{0}}{2.405 \gamma v e b^{*}} .
$$

At $T_{0} \simeq 10 \mathrm{~K}, v \sim 10^{7} \mathrm{~cm} / \mathrm{s}, b^{*} \simeq 7.7 \AA$ the right-hand side of (12) would yield $H_{1} \lesssim 10^{5}$ Oe.

At larger $H$, according to $\left(10^{\prime}\right)$, the SDW phase is completely restored with $T_{\text {SDw }}=T_{0}$, which could be even higher than $T_{\mathrm{SDw}}$ at $P=0$, since, of course, $t_{b^{*}}^{\prime}$ is finite at ambient pressure. When the magnetic field decreases, the " points" of the metallic phase (at $T=0)$ are concentrated near $H=0$ according to the asymptotic behaviour of the Bessel function zeroes (11') :

$$
2 c t_{b^{*}}^{\prime} / v e H_{k} b^{*} \simeq \pi\left(k-\frac{1}{4}\right), \quad(k \gg 1) .
$$

A schematic $T-H$ phase diagram at fixed pressure (i.e. at fixed $t_{b^{*}}^{\prime}$ ) is shown in figure 2 . The typical « transition temperature » $T_{\mathrm{SDw}}(k)$, the value of the « subphase » $k$, exponentially decreases with an increase of the number $k$. With logarithmic accuracy the corresponding behaviour can be obtained directly from equation $\left(10^{\prime}\right)$ :

$$
\ln \frac{\pi T_{0}}{2 t_{b^{*}}^{\prime} \gamma} \simeq-J_{0}^{2}\left(\frac{2 c t_{b^{*}}^{\prime}}{v e H b^{*}}\right) \ln \frac{b^{*} e H v}{c T_{\mathrm{SDW}}} .
$$

This infinite sequence of transitions would resemble to some extent a sequence of exciton phases in semiconductors, the possibility of which was discussed in [12] many years ago. The phase diagram of the type shown in figure 2 is, of course, an artefact of the 2D dispersion law, (see below) and becomes unstable if some finite $t_{c^{*}}^{\prime}$ is taken into account. In fact, according to (10) the presence of the factor $J_{0}\left(\frac{4 t_{c^{*}}^{\prime} x}{v}\right)$ in the integral immediately makes again the whole integral convergent at large $x \sim v / t_{c^{*}}^{\prime}$. Comparing the value of $T_{\mathrm{SDw}}$ estimated at small $H$ from equation (13) with the value of $t_{c^{*}}^{\prime}$, one gets that the oscillations shown in figure 2 do appear only at the onset field $H>H_{0}$ where

$$
H_{0} \simeq \frac{\pi c t_{b^{*}}^{\prime}}{v e b^{*}} \ln \frac{\pi T_{0}}{2 t_{b^{*}}^{\prime} \gamma} / \ln \frac{t_{c^{*}}^{\prime}}{t_{b^{*}}^{\prime}}
$$

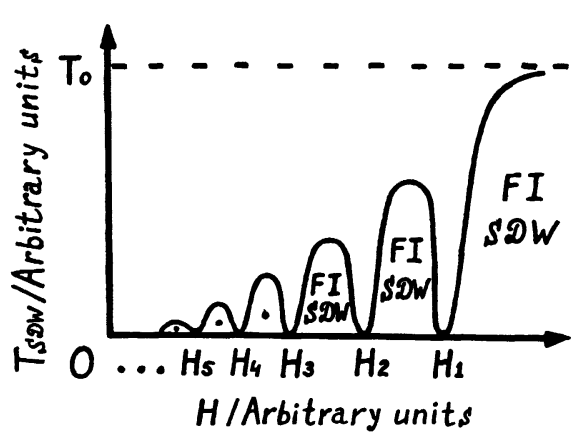

Fig. 2. - Infinite series of possible phase transitions in the presence of the magnetic field $H$. 
and only a few oscillations can be realized in practice. Let us also add that the finite $t_{c^{*}}^{\prime}$ converts the "points " of the metallic phase stability, $H_{k}$, into small stability " islands ", the width of which can again be estimated from the condition :

$$
J_{0}^{2}\left(\frac{2 c t_{b^{*}}^{\prime}}{v e H b^{*}}\right) \ln \frac{b^{*} e H v}{c t_{c^{*}}^{\prime}} \sim 1
$$

for the magnetic fields in the vicinity of the corresponding $H_{k}$ from $\left(11^{\prime}\right)$.

At this stage, we shall not go into further details, since our stability condition (10) holds for both the thermodynamic stability of the phase and its metastability. Otherwise, the boundary line in figure 2 , in principle, could describe the "supercooling " line for the metallic phase while the real transition into the SDW phase (or between two different SDW phases) would be of the first order. Some hysteresis features have been observed in [4] near some field values ascribed to the $\mathrm{SdH}$ oscillations. The sharp temperature-dependent steps in the Hall voltage in (TMTSF $)_{2} \mathrm{ClO}_{4}$ [7] also point in the same direction. Note that the magnetic field appears in the discussed phenomenon approximately in the same combination as would be also expected in the SdH effect definition of the closed orbit areas for the hypothetic semimetallic SDW phase (cf. Eqs. (10) and $\left(3^{\prime}\right)$ ). As for $t_{\perp}^{\prime}\left(\mathbf{p}_{\perp}\right)$, it has been assumed in the form of equation $\left(3^{\prime}\right)$ only for the sake of convenience for the subsequent discussion. Its modification does not change the precedent results qualitatively.

We would also like to repeat that at the field $H>H_{1}$ from equation (12) the SDW finally becomes fixed. Since the above discussion shows that the dielectric state emerges in a large field (at $T=0$ ), all the conducting mechanisms are expected to disappear. However a more thorough investigation of the SDW phases, restored by the magnetic field, is necessary.

Unlike the SDW phase, the CDW state in general is suppressed by a strong magnetic field, according to equation $\left(1^{\prime}\right)$. Nevertheless, the competition between this inhibiting mechanism and the phenomenon of the " one-dimensionalization " of the electron spectrum expressed by equation (10), could be important also for the CDW pairing at the intermediate fields because both mechanisms involve the same dimensionless combination of parameters.

So far we have preferred to explain the phenomenon in terms of the nesting vector from equation (4), since most of new effects observed, including the unusual « SDW » oscillations, may be described in this case by the appropriate choice of $T_{0}$ and $t_{\perp}^{\prime}$. The main exception is that the sizes of the electron (hole) pockets, as defined by the Hall measurements [7, 8, 13] in (TMTSF) $)_{2} \mathrm{ClO}_{4}$, are too large. This gives rise again to the necessity of discussing the pairing with the "direct " nesting vector $\left(2 k_{\mathrm{F}}, 0,0\right)$. There are two points which should be mentioned in this connection.

For the 3D (or 2D) electron dispersion law electron-electron interactions restore the Fermi liquid description, if the temperature is low enough. The above results (Eqs. $(8,9))$ state rigorously that the $2 \mathrm{D}$ electron spectrum, if its Fermi surface consists of two separate open sheets, is absolutely unstable at a low enough temperature (at the appropriate sign of interaction) with respect to the SDW electron-hole pairing. The physics is that electron and hole from the opposite sides of the Fermi surface move together along the chain axis, their motion being limited in space in transverse direction, provided the magnetic field is imposed.

For the (TMTSF) ${ }_{2} \mathrm{X}$ one-chain-compounds it is plausible to consider equation (3) as a good approximation for their electron spectrum. In this case in all the expressions one should substitute $t_{b^{*}, c^{*}}\left(\right.$ and $\left.b^{*} / 2, c^{*} / 2\right)$ for $t_{b^{*}, c^{*}}^{\prime}$ (and $\left.b^{*}, c^{*}\right)$ in accordance with (3). It appears now that the instability mentioned above is related to a wider class of the nesting wave vectors

$$
\mathbf{Q}=\left(2 k_{\mathrm{F}}+\delta, 0,0\right)
$$


and, instead of equation $\left(10^{\prime}\right)$, one has

$$
\frac{1}{\zeta}-\int_{d}^{\infty} J_{0}\left[\frac{8 c t_{b^{*}}}{e H v b^{*}} \sin \left(\frac{e H b^{*}}{2 c} x\right)\right] \cos (\delta x) \frac{2 \pi T \mathrm{~d} x}{v \operatorname{sh} \frac{2 \pi T x}{v}} \geqslant 0 .
$$

Using the same procedure of the averaging of the oscillating factors over $x$ at $T \rightarrow 0$, one obtains easily that the logarithmic divergence at large $x$ is now connected with the coefficient :

$$
J_{m}^{2}\left(\frac{4 c t_{b^{*}}}{v e H b^{*}}\right) ; \quad m=\frac{\delta c}{e H b^{*}} .
$$

This coefficient reaches its maximum at

$$
m \simeq \frac{4 c t_{b^{*}}}{v e H b^{*}} ; \quad \delta=\frac{4 t_{b^{*}}}{v} .
$$

In other words, at low fields the instability would for the first time correspond to the biggest possible electron (or hole) pockets. The size of pockets given by (14) is in a good correspondence with the $t_{b^{*}}$ estimates $\left(t_{b^{*}} \sim 150 \mathrm{~K}\right)$ and the Hall effect data [7, 8]. The possibility to construct the ground states of a set of subphases characterized by $\delta$ which decreases with an increase in magnetic field, is now under investigation.

In conclusion, we have given theoretical arguments explaining the puzzling fact of restoration of the SDW phase in the (TMTSF) ${ }_{2} \mathrm{X}$ compounds by the magnetic field. The rôle of fluctuations of any kind has not been considered, since temperatures are assumed to be low enough to permit the Fermi liquid approach. Two models (i.e. the " perfect " nesting and the " direct ", or onedimensional pairing) could correspond to the physical situation in different compounds. The Hall data $[7,8]$ for $\mathrm{ClO}_{4}$ showing comparatively large pocket volume in this material might be understood in terms of such a direct nesting. The expected phase diagram is shown schematically in figure 3.

\section{Acknowledgments.}

One of us (L. G.) is grateful to D. Jérome who has first drawn his attention to the phase diagram of these materials and to S. Barisič for stimulating discussions.

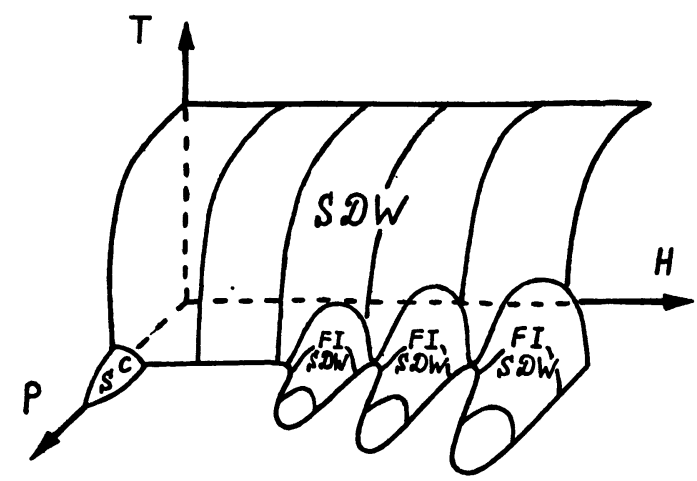

Fig. 3. - The phase diagram of Fig. 1 with the theoretical representations, developed in the text, taken into account. 


\section{References}

[1] Kwak, J., J. Physique Colloq. 44 (1983) C3-839.

[2] Brusetti, R., Garoche, P. and Bechgaard, K., J. Physique Colloq. (1983) C3-1051.

[3] Kwak, J., Schirber, J., Green, R. and Engler, E., Phys. Rev. Lett. 46 (1981) 1296.

[4] Kajimura, K., Tokumoto, H., Tokumoto, M., Murata, K., Ukachi, T., Anzai, H., Ishiguro, T. and Saito, G., J. Physique Colloq. 44 (1983) C3-1059.

[5] Azevedo, L., Schirber, J., Green, R. and Engler, E., Physica B 108 (1981) 1183.

[6] Takahashi, T., Jerome, D. and Bechgaard, K., J. Physique Lett. 43 (1982) L-565.

[7] Ribault, M., JÉrome, D., Tuchendler, J., Weyl, C. and BeChgaARd, K., J. Physique Lett. 44 (1983) L-953.

[8] Chaikin, P. M., Mu-Yong Choi, Kwak, J. F., Brooks, J. S., Martin, K. P., Naughton, M. J., Engler, E. M. and Greene, R. L., Phys. Rev. Lett. 51 (1983) 2333.

[9] Keldysh, L. and Kopaev, Yu., Fiz. Tverd. Tela 6 (1964) 2791.

[10] Horovitz, B., Gutfreund, H. and Weger, M., Phys. Rev. B 12 (1975) 3174.

[11] Jerome, D., J. Physique Colloq. 44 (1983) C3-775.

[12] KoHn, W., Phys. Rev. Lett. 19 (1967) 439.

[13] Kwak, J. F., Phys. Rev. B 28 (1983) 3277. 